\title{
A Systematic Mapping Study of Cloud E-Learning Computing for Education
}

\author{
Faiz Akram \\ Department of Information Technology \\ Shri Venkateshwara University \\ Gajraula, Uttar Pradesh, India
}

\author{
Rajeev Kumar, PhD \\ Faculty of Engineering and Technology \\ Shri Venkateshwara University \\ Gajraula, Uttar Pradesh, India
}

\begin{abstract}
This paper examines the cloud computing for education (CCE) literature, and analyzes if the research is developing scientifically with adequate empirical validation. All aspects of empirical investigations covered in the literature are shown as weak, hence, the necessary scientific development of CCE requires extending its scope of interest, and involving scholars synergistically to create and maintain a "common research agenda". Differences are found across geographic areas in applying CCE infrastructure and technologies in educational institutions; few studies address CCE's impact on pedagogic processes. The scope of interest in CCE is only partially covered; with empirical research being very shallow. Suggestions are made for more effective research on concerning the production and use of content.
\end{abstract}

\section{Keywords}

Cloud E-learning, computing for Education, Electronic Learning

\section{INTRODUCTION}

E-LEARNING (electronic learning) is a dynamic field in the connected software engineering space, advanced by late data innovation developments. A more typical term is Technology Enhanced Learning (TEL) [1]; recently rose advancements have prompted new types of TEL, for example, m-learning (portable taking in), an administration that ordinarily joins versatile and remote advances to give learning instruments and instructive assets, making lessons accessible and available to anybody wherever through cell phones [2]. Another case of TEL is vlearning (virtual getting the hang of), giving rich learning assets in an extraordinary, effectively open virtual condition [3]. Elearning administrations make utilization of concentrated registering situations (e.g., virtual universes, reenactments, video spilling, information investigation, exact experimentation) and largescale participation for content creation, for example, Massive Open Online Courses (MOOC). Distributed computing is a model for empowering advantageous, on request organize access to a mutual pool of configurable figuring assets, (for example, systems, servers, stockpiling, applications and administrations) that can be quickly provisioned and discharged with at least administration exertion and connection with the provider [4], [5].

In view of [5], distributed computing should exhibit five fundamental qualities: (1) on-request self-benefit, enabling customers to deal with their own particular virtual assets without interfacing with the provider, with expansive system get to (e.g., the Internet) by means of heterogeneous customer stages, for example, cell phones, tablets and PCs; (2) asset pooling, making assets available to numerous makers and to any approved buyer; (3) fast allotment, giving assets rapidly and discharging them promptly after utilize; (4) estimated benefit, naturally controlling the arrangement of administrations and (5) metric layouts to enhance and measure (as far as perceivability and installment) benefit arrangement for both the provider and the client. These five highlights give numerous open doors in instructive improvement [6]. Distributed computing can give a few fascinating instruments to the two educators and understudies, for instance, showing processing assets for lessons and labs on ask for and as per diverse client needs. Distributed computing encourages the arrangement of more adaptable courses in light of understudies' particular needs, when and where they need; it additionally enables educators to make exercise content that meets their particular necessities [3]. The cost of such assets relies upon the amount and time of utilization.

For instance, where educators need to make a virtual registering condition (virtual machine) for a lab exercise, a few foundations are utilizing distributed computing's joint effort and capacity administrations to make a virtual learning condition [3] that would be costly and tedious, all things considered. Distributed computing encourages the boundless utilization of TEL, without requirement for framework or particular gifted faculty, and inside a short measure of time.

\section{RELATIVE WORK}

A systematic study of the writing is an optional report technique that has developed in the course of the most recent two decades, and that can be viewed as an advancement of research in view of essential investigations of exact confirmation [1]. One of the main efficient overviews was in solution, a teach that is especially put resources into prove based research. This all the more as of late enlivened the exchange of the requirement for, and idea of, a precise overview to the area of data frameworks [2] and, in parallel, programming building [3]. Pickard et al. [4] examine the need to total the aftereffects of experimental essential examinations to frame an optional report. Mill operator [5] addresses the issue of joining essential examination comes about through meta-investigation, and Hayes presents the idea of research amalgamation. They demand the significance of precise essential investigations to fabricate learning by incorporating past outcomes. A first endeavor at a combination of essential investigations was completed by Basili et al. [6] CCE is an interdisciplinary research territory including the fields of software engineering and teaching method [7], the two trains that can profit by look into comes about in light of experimental confirmation. The consequences of this present investigation could consequently profit the two groups. Given the moderately youthful age of this examination zone, the creators think of it as suitable to begin with a SMS, since if the outcomes demonstrate restricted or off base, it won't serve to play out any combination, as recommended in the references refered to. For instance, a SLR paper [8] in CCE exhibits moderately couple of productions applicable for consideration in the investigation; its discoveries are a subset of those resultant from a SMS, when all is said in done, and it express 
that it needs exact proof, and in this way can't lead the metaexamination unmistakable of the SLR. To the creators' learning, there are only two past writing audits on the theme. The principal consider by Fasihuddin et al. [6] examines the best in class of the examination without considering the exact proof factor. Since this investigation did not take after a precise approach, the repeatability and unwavering quality [10] are low, and it meets the objective of the examination displayed here. The second investigation by Gonzàlez-Martìnez et al. is a SMS that gives intriguing outcomes on the preferences and constraints of the utilization of CCE. In spite of the fact that guaranteeing to utilize the rules in [11], it doesn't generally fit in with them, for instance, assessing the nature of papers as is run of the mill of a SLR. In addition, with the quality estimation parameter as the "pertinence" of the commitment for the instructive space, "validity," "soundness," and "clearness" are not determined; these measures are then subjectively assessed by the scientists, so the examination isn't replicable. It plots the cutting edge however does not break down to what degree the outcomes depend on exact proof. The examination exhibited here covers the arrangement of some exploration themes, and varies from the papers refered to beforehand in numerous different angles.

\section{CLOUD E-LEARNING}

Cloud e-Learning (CeL) is a new worldview for e-learning in which students are given a naturally produced learning way that uses any appropriate sources from the cloud [1]. CeL is considered as a progression of e-learning and intends to give customized administrations that will expand collaboration between clients who share a pool of encounters and information. CeL ought to recommend organized courses that match students' inclinations and subjective level. The Learning Cloud involves distinctive hotspots for CeL and everything put away in it can conceivably be utilized for learning purposes.

The fundamental objective is to naturally produce a customized learning way of learning objects that sensibly meets the profile and wants of the student. Prior to any personalization is even viewed as, the fundamental issue CeL needs to address is the heterogeneity of electronic assets that frame the Learning Objects (LOs). Competitor LOs experience the ill effects of: (a) no or little semantics/explanation, (b) assortment of granularity, and (c) no methods for sticking them together in versatile request to make an intelligible course. Such learning materials can barely fit together [2] in a sensible learning way in light of their distinctive measures. For example, a LO may not fit with another LO straightforwardly, as a result of various metadata principles or diverse learning objects norms or conflicting planned learning results and wanted psychological level.

In $\mathrm{CeL}$, we visualize a procedure that takes these unstructured learning materials and adjusts them for having the capacity to make a sound grouping. In current e-learning approaches, organized LOs are put away in vaults (LORs) and they can be utilized inside the setting of their stores to make customized learning ways. Despite what might be expected, in CeL, the heterogeneity of unstructured or semi-organized electronic sources makes tweaked taking in a testing errand.

\section{METHODOLOGY}

This research strategy applies the rules of Kitchenham and Chartres to lead the SMS. A.

Research Questions Based on Kitchenham's article, the goal of the present SMS utilizes the worldview: Population, Intervention, and Output (PIO). Kitchenham and Chartres recommended utilizing a "Correlation" factor, rejected here on the grounds that, in a SMS, the papers' substance are just halfway broke down. The papers' creators scarcely clarify if and how they completed the examination, so choice of these papers in light of such criteria can't be made. For particular goals, the PIO worldview is characterized as: Population: specialists, educators and beneficiaries in instruction of all levels; proceeding with training laborers inspired by utilizing distributed computing; and academic establishments;

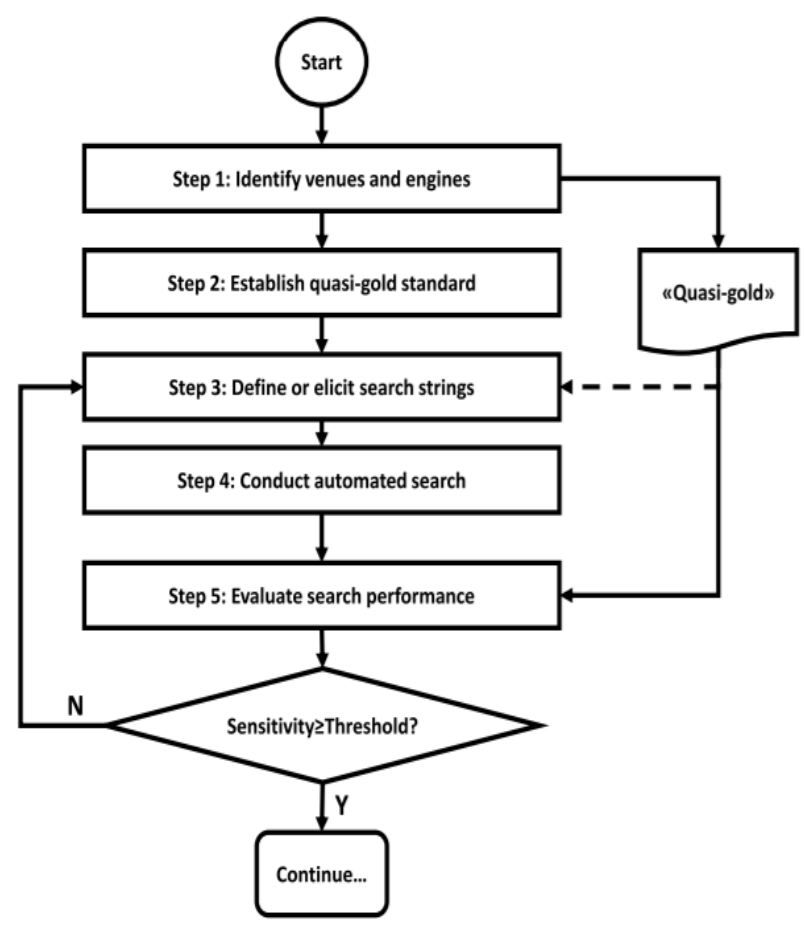

Figure 1: Validation of the research string

Intervention: cloud computing during the entire educational lifecycle; Output: any benefits that cloud computing can lead to in terms of organizations, teaching methods and teaching processes.

B. Research Protocol 1) Research String: To construct an exploration string that naturally guarantees the extraction of an applicable writing study test with the end goal of this paper, one must know the words utilized as a part of the writing that express the ideas in the PIO. These words, incrementally got through the approval procedure depicted in the following section, are:

1) Population: teacher; educator; learner; student; teaching; education; learning; education courses; educational courses; distance learning; computer-aided instruction; education institution; e-learning; electronic learning; learning management system; distance education; m-learning; v-learning; technologyenhanced learning; TEL; massive open online courses; MOOC; virtual machine; virtual campus; virtual learning environments; VLE.

2) Intervention: cloud computing; cloud.

3) Output: enhancing educating; educational changes; worldwide instructing; adapting anyplace; learning whenever; educating anyplace; instructing whenever. The inquiry string is comprised of words or measurements connected to "OR" and words or measurements connected to "AND". 2) Research Strategy: Before playing out a programmed extraction over the whole timeframe considered, an inquiry string must be manufactured that guarantees the extraction of delegate 
contemplates in the whole writing concerning CCE. To do this, the procedure proposed by Zhang et al. [13], Fig. 1, was utilized.

4) Randomly Assigning Papers to Author Reviewers: The authors of this paper assumed the roles of reviewers, evaluating each selected publication with respect to the inclusion or exclusion criteria and with respect to the keyword extraction. Each paper received three reviews, and each of the authors was randomly assigned the same number of papers to revise. In total, each author reviewed 348 publications.

5) Screening Significant Papers for Inclusion and Exclusion: The screening procedure announced the most fitting papers for the mapping study through the consideration and avoidance criteria gave by the convention. In this manner, diary papers, meeting and co-found occasion distributions and specialized reports were incorporated. At the point when different productions managed a similar theme, the latest ones were taken. The dialect considered was English. The information gathering period was January 2012 - December 2016. Concentrates that did not plainly report results and papers accessible just through edited compositions or introductions were barred; examines applicable to distributed computing yet without any references to training were likewise disposed of. For each paper, the three analysts communicated their assessments for incorporation or avoidance. On the off chance that they dissented, they talked about until achieving assention. In the event that no understanding was accomplished inside as far as possible (30 minutes), the paper was acknowledged or dismissed by greater part.

\section{LO GRANULARITY AND INDENTED LEARNING OUTCOMES}

One of the most important characteristics of LOs that are aiming to be shared and reused is the granularity of the content [9]. Granularity alludes to the learning time required to commit to a given LO and it is corresponding to the span of the LO. Cases incorporate video length, the quantity of pages in a book, or the exertion allotted to finish an errand, for example, a composed task, a test, and so on. The granularity of a LO could have an impact when incorporating distinctive substance models into a solitary and reasonable grouping of LOs or when endeavoring to reuse the same LO in various settings. Reusability is one of the key qualities of LOs. Granularity of a LO is characterized distinctively by various associations and inside various Content Models (SCORM, CISCO, Learnativity, IMS Content Packaging and so forth.) [11].

TABLE I: Revised Bloom's Taxonomy

\begin{tabular}{|l||l|}
\hline Pyramid Levels & Key Verbs (keywords) \\
\hline \hline Creating & $\begin{array}{l}\text { design, formulate, build, invent, create, compose, } \\
\text { generate, derive, modify, develop }\end{array}$ \\
\hline Evaluating & $\begin{array}{l}\text { choose, support, relate, determine, defend, judge, } \\
\text { grade, compare, contrast, argue, justify, support, } \\
\text { convince, select, evaluate. }\end{array}$ \\
\hline Analyzing & $\begin{array}{l}\text { classify, break down, categorise, analyze, diagram, } \\
\text { illustrate, criticise, simplify, associate. }\end{array}$ \\
\hline Applying & $\begin{array}{l}\text { calculate, predict, apply, solve, illustrate, use, } \\
\text { demonstrate, determine, model, perform, present. }\end{array}$ \\
\hline Understanding & $\begin{array}{l}\text { describe, explain, paraphrase, restate, give original } \\
\text { examples of, summarise, contrast,interpret, discuss. }\end{array}$ \\
\hline Remembering & $\begin{array}{l}\text { list, recite, outline, define, name, match, quote, } \\
\text { recall, identify, label, recognise }\end{array}$ \\
\hline
\end{tabular}

\section{CONCLUSION}

This mapping study identified significant interest in CCE from the high number of papers in the literature over the three year period analyzed. Research in CCE is creating in accordance with investigate in distributed computing, however a critical advance would be for CCE to develop more in building up nations' colleges and higher instructive organizations, particularly on points of general premium identified with distributed computing (e.g., cost sparing, security, superior). The extent of enthusiasm for CCE in the writing primarily centers around the mechanical angles, rather than on the effect of distributed computing on instructional method and the advancements that it could convey to educating and learning forms.

\section{REFERENCES}

[1] Krenare Pireva, Petros Kefalas and Ioanna Stamatopoulou, "Representation of learning objects in cloud e-learning", Information, Intelligence, Systems \& Applications (IISA), 8th International Conference on, IEEE 2017.

[2] E.-M. Kalogeraki, C. Troussas, D. Apostolou, M. Virvou, T. Panayiotopoulos, "Ontology-based model for learning object metadata", Information Intelligence Systems \& Applications (IISA) 2016 7th International Conference on. IEEE, pp. 1-6, 2016.

[3] Cubillo, J., Marten, S. \& Castro, M. (2011) ' New Technologies Applied in the Educational Process', IEEE Global Engineering Education Conference (EDUCON) Learning Environments and Ecosystems in Engineering Education, April 4 - 6, (2010) Amman, Jordan, pp. 575584.

[4] Armbrust, M., Fox, A., Griffith, R., Joseph, A. D., Katz, R. H., Konwinski, A., Lee, G., Patterson, D. A., Rabkin, A., Stoica, I. \& Zaharia, M. (2009) Above the Clouds: A Berkeley View of Cloud Computing, Electrical Engineering and Computer Sciences University of California at Berkeley.

[5] Angad Grewal, Shri Rai, Rob Phillips and Chun Che Fung "The E-Learning Lifecycle and its Services: The Web Services Approach" Proceedings of the Second International Conference on eLearning for KnowledgeBased Society, August 4-7, 2005.

[6] J. A. Gonzàlez-Martìnez, M. L. Bote-Lorenzo, E. GòmezSànchez, and R. Cano-Parra, "Cloud Computing and education: A state-of-the-art survey," Comput. Educ., vol. 80, pp. 132-151, Jan. 2015.

[7] M. Joshi, "Emerging trends of cloud computing in education methodologies: A review," Int. J. Adv. Res. Comput. Sci. Softw. Eng., vol. 5, no. 12, pp. 1-4, Dec. 2015.

[8] M. S. Ibrahim, N. Salleh, and S. Misra, "Empirical studies of cloud computing in education: A systematic literature review," in Computational Science and Its ApplicationsICCSA 2015 (LNCS 9158), O. Gervasi et al., Eds. Cham, Switzerland: Springer, 2015.

[9] R. Boyatt and J. Sinclair, "Navigating the educational cloud," in Proc. Workshop Learn. Technol. Educ. Cloud, vol. 173, 2012, pp. 179-191.

[10] M. S. Ibrahim, N. Salleh, and S. Misra, "Empirical studies of cloud computing in education: A systematic literature review," in Proc. Int. Conf. Comput. Sci. Appl., Jun. 2015, pp. 725-737.

[11] B. Kitchenham and S. Chartres, "Guidelines for performing systematic literature reviews in software engineering," Keele Univ., Newcastle, U.K., and Durham Univ., Durham, U.K., Rep. EBSE-2007-01, 2007. 
[12] R. Wieringa, N. Maiden, N. Mead, and C. Rolland, "Requirements engineering paper classification and evaluation criteria: A proposal and a discussion," Requirement Eng., vol. 11, no. 1, pp. 102-107, 2006.

[13] D. I. K. Sjoberg, T. Dybå, and M. Jørgensen, "The future of empirical methods in software engineering research," in Proc. IEEE Future Softw. Eng. (FOSE), May 2007, pp. $358-378$

[14] N. Sclater, "Cloud computing in education," UNESCO Inst. Inf. Technol. Educ., Moscow, Russia, Rep. IITE/INS/PB/2010/01, 2010.
[15] B. Kitchenham, P. Brereton, Z. Li, D. Budgen, and A Burn, "Repeatability of systematic literature reviews," in Proc. Int. Conf. Eval. Assess. Softw. Eng. (EASE), Durham, U.K., 2011, pp. 46-55.

[16] S. MacDonell, M. Shepperd, B. Kitchenham, and E. Mendes, "How reliable are systematic reviews in empirical software engineering?" IEEE Trans. Softw. Eng., vol. 36, no. 5, pp. 676-687, Sep./Oct. 2010. 